\title{
"Les aspects idéologiques dans "La Répudiation" de Rachid
}

\section{Boudjedra"}

\author{
Ibrahim Abouelmaati Ibrahim Elmorsy \\ dr.ibrahimmaati@gmail.com
}

Maître de conférences à la Faculté des Lettres -Université de

Kafrelsheikh

\section{Résumé :}

Rachid Boudjedra et son premier ouvrage romanesque "La

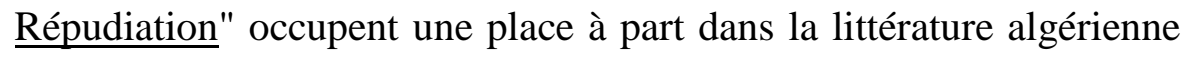
d'expression française. La problématique de notre recherche est basée sur son premier roman "La Répudiation" dont le thème dominant touche presque toutes les familles : c'est celui de la répudiation. Il est clair que le titre du roman s'accorde bien à son contenu.

Notre recherche porte comme titre :" Les aspects idéologiques dans "La Répudiation" de Rachid Boudjedra." Nous pénétrons dans les profondeurs de la pensée de cet écrivain. A travers notre travail, nous traitons l'aspect politique chez Boudjedra, bien présent tout au long du roman, en affirmant que la littérature et la politique ne se séparent pas. Nous analysons également l'aspect érotique; à travers lequel, l'écrivain traite les relations taboues et incestueuses entre les personnages. Le tabou chez Boudjedra s'est étendu pour frapper la plupart des personnages du roman dont la relation était énigmatique. L'aspect social occupe aussi une place distinguée où le romancier traite la souffrance de toutes les femmes algériennes en insistant sur quelques phénomènes sociaux comme le remariage du père, la mendicité et la sorcellerie...etc.

L'aspect religieux n'était pas absent de l'idéologie boudjedrienne qui considère que le statut de la femme en Islam est mineur face à celui de l'homme privilégié, en passant par d'autres thèmes comme le mois du Ramadhan et tout ce qui se rattache à ce moins sacré. Enfin, Boudjedra jette quelques lumières sur l'importance des marabouts chez les femmes algériennes. 
L'aspect romanesque au service de l'idéologie boudjedrienne termine notre travail ; "La Répudiation", n'a pas réussi seulement à présenter une structure romanesque bien différente; mais il nous fascine aussi par son écriture singulière et l'influence de Boudjedra par d'autres romanciers. Nous dévoilons finalement le thème de son choix de la langue de l'Autre.

En bref, "La Répudiation", en tant qu'un ouvrage romanesque mêlant le réel à la fiction, a bien exprimé tout ce qui se déroule dans la mentalité de Rachid Boudjedra non pas seulement comme un écrivain d'expression française, mais aussi comme un homme arabomusulman.

Mots clés : Idéologie, l'aspect romanesque, l'aspect politique, l'aspect érotique, l'aspect social, l'aspect religieux.

Rachid Boudjedra est considéré comme l'un des plus grands romanciers maghrébins d'expression française, très connu autant dans son pays d'origine qu'à l'étranger. Il faudrait signaler le bilinguisme romanesque de cet écrivain qui appartient à la fois à deux genres de littérature : la littérature algérienne d'expression française d'une part, et la littérature algérienne d'expression arabe de l'autre part; c'est un bilinguisme français-arabe. Boudjedra se distingue par sa maîtrise de diverses langues, il est le polyglotte qui ne trouve aucune difficulté avec ses langues, Armelle Crouzières-Ingenthron qualifie ce multilingue en disant: "Jongleur de mots, il superpose des textes en arabe, en berbère, en français, en espagnol et en anglais dans La Prise de

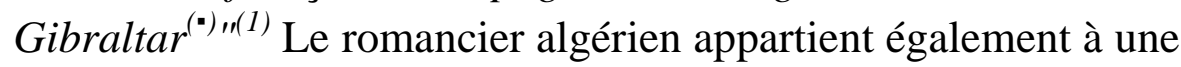
nouvelle génération "dont la principale caractéristique demeure la critique sans complaisance des valeurs datées, surannées $d u$ monde arabo-musulman, $d u$ pouvoir algérien post-

(") Boudjedra (Rachid), roman traduit de l'arabe par Antoine Moussali en collaboration avec l'auteur, éditions Denoël, Paris, 1987.

$\left(^{1}\right)$ Crouzières-Ingenthron (Armelle), Le double pluriel dans les romans de Rachid Boudjedra, L'Harmattan, paris, 2001, p.125 
indépendantiste. ${ }^{\prime(2)} \mathrm{Sa}$ fresque littéraire occupe une place singulière; Hafid Gafaïti apprécie que l'œuvre de Boudjedra "se caractérise par une double subversion, sur le plan thématique et discursif et sur celui de l'écriture." (3) ; Passons maintenant à l'importance et la valeur de notre corpus, titré " $\underline{\text { La }}$ Répudiation", récit très touffu d'idées, qui donne bien une expression spéciale de la pensée de son auteur. Armelle Crouzières-Ingenthron apprécie que :" La critique est unanime quant à l'importance et la place unique de ce premier roman au sein de la littérature algérienne." ${ }^{(4)}$

À première vue, le titre du roman nous pousse à plonger dans l'univers spécifique à chaque femme répudiée; le titre nous amène à s'interroger sur les causes et les conséquences de cette répudiation. Un roman d'expression française daté 1969, c'est-àdire sept ans après l'indépendance de l'Algérie, pose plusieurs questions concernant l'auteur et son œuvre : pourquoi la femme algérienne et surtout la répudiée souffre toujours? Le pays est encore sous le poids de la colonisation ? Pourquoi Boudjedra at-il choisi la langue de l'autre pour s'exprimer? Les idées de l'auteur, traduites à travers les événements du roman, reflètent une part réelle ou fictive de l'Histoire de son pays ? Hafid Gafaiti constate que: "La Répudiation, son premier roman, constitue une date charnière, par son effet de rupture, pour la littérature algérienne, dans la mesure où il brisait les tabous

(2) Alemdjrodo (Kangni), Rachid Boudjedra, la passion de l'intertexte, éd. Presses Universitaires de Bordeaux, 2001, disponible sur le site "https://books.openedition.org/pub/3086\#text"

(3) Gafaïti (Hafid), Rachid Boudjedra, une poétique de la subversion, Autobiographie et Histoire, éd. L'Harmattan, Paris, 1999, p.37

(4) Crouzières-Ingenthron (Armelle), Le double pluriel dans les romans de Rachid Boudjedra, Op. Cit., p.19 
essentiels que sa production ne cessera, esthétiquement, d'exorciser: la politique, la sexualité et la religion."

Armelle Crouzières-Ingenthron nous donne une autre interprétation de l'écriture de Boudjedra d'un roman portant un titre comme ça: "La Répudiation", il apprécie que :" pour retrouver la mère, la faire exister à nouveau, lui redonner vie par l'écriture puisque selon lui, elle n'a jamais existé, et cela encore moins après sa répudiation." ${ }^{\prime(6)}$

Notre problématique est basée sur les aspects idéologiques dans le premier ouvrage romanesque de Rachid Boudjedra: "La Répudiation". Pour cette raison, il est très nécessaire de donner une définition claire du mot "idéologie", sur lequel va être concentrée notre recherche. D'après le Centre National de Ressources Textuelles et Lexicales (CNRTL), le mot "idéologie" signifie "Ensemble plus ou moins cohérent des idées, des croyances et des doctrines philosophiques, religieuses, politiques, économiques, sociales, propre à une époque, une société, une classe et qui oriente l'action"(7); et le mot signifie aussi : "Ensemble de croyances, des idées caractéristiques d'une personne, d'un groupe, d'une société à un moment donné."(8) Ainsi le terme "idéologie" se rattache aux idées, aux doctrines et aux croyances d'une personne ou d'une société.

Notre étude découvre l'itinéraire d'idées concernant un écrivain arabe de nationalité algérienne et de langue française à travers son roman "La Répudiation" dont la structure, le style et les

(5) Gafaïti (Hafid), Rachid Boudjedra, une poétique de la subversion, Autobiographie et Histoire, Op. Cit., pp.37-38

(6)Crouzières-Ingenthron (Armelle), Le double pluriel dans les romans de Rachid Boudjedra, Op. Cit., p.125

(7) https://www.cnrtl.fr/definition/id\%C3\%A9ologie

(8) https://www.linternaute.fr/dictionnaire/fr/definition/ideologie/ 
thèmes abordés nous emmènent à rechercher profondément l'idéologie et peut-être l'être de cet écrivain.

Hafid Gafaïti apprécie que :" Ce qui caractérise justement l'entreprise de Boudjedra, c'est la tentative consciente de l'écrivain de fonder son identité sur la base de son æeuvre. ${ }^{\prime(9)}$; Boudjedra, lui-même, donne à son lecteur une analyse de contenu du roman dans quelques mots à la fois simples et profondes, portant beaucoup de détails et d'éclaircissements sur ce que présente cet auteur mal compris lors de la parution du roman : " Dans La Répudiation je voulais non pas témoigner mais représenter une certaine réalité profonde intérieure, poétique de l'Algérie d'avant et d'après l'indépendance.(...)Un texte sur l'Algérie ou simplement une autobiographie est un prétexte à écrire, à poétiser, finalement, à désamorcer le réel." ${ }^{\prime 10)}$.

Ainsi, "La Répudiation" représente un amalgame des idées exprimant tout ce qui se déroule dans la tête d'un homme recherchant le mieux pour son pays, et en espérant trouver son identité perdue du fait des conquêtes successives et de mentalité stérile de la société patriarcale.

\section{L'aspect politique :}

Dans la totalité de son œuvre romanesque, Boudjedra est très soucieux des événements historiques rattachant à l'Histoire de son pays qui le poursuit d'un roman à l'autre. En s'intéressant le plus souvent à découvrir les événements bien reliés à la période qui précède et qui succède à l'indépendance, le romancier les met en scène pour mettre au jour une réalité cachée du système

(9) Gafaïti (Hafid), Rachid Boudjedra, une poétique de la subversion, Autobiographie et Histoire, Op. Cit., p.11

(10) Boudjedra (Rachid), in Présence Francophone, No. 19, Automne, 1979, pp.61-62 
politique établi. Boudjedra n'était pas à l'écart des problèmes de l'Algérie ; son enthousiasme, voire son patriotisme le poussent à prendre parti non seulement par son discours, mais aussi par l'action. Hafid Gafaïti souligne que l'engagement de Boudjedra: " se manifeste par la participation à des actions concrètes telles que les manifestations, son activité au sein de la Ligue Algérienne des Droits de l'Homme ainsi que d'autres organisations politiques, des débats et des interventions publiques autant en Algérie qu'à l'étranger." (11)

Ainsi, on constate la relation étroite entre la littérature et les événements politiques dominants; l'écrivain, par sa plume, essaie toujours de dévoiler la réalité vécue de son peuple ; en mêlant le réel à la fiction, l'auteur donne l'essentiel de sa pensée. Guy Dugas considère l'année 1945 comme le début de : "la littérature maghrébine de langue française en quantité et qualité" en parallèle avec l'émergence du "mouvement nationaliste". (12)

Boudjedra exprime ses points de vue, plutôt son expérience déjà vécue, en ce qui concerne la relation franco-algérienne en soulignant la mésentente entre les deux pôles. L'incipit du roman expose bien ses idées sur cette relation non terminée avec l'ex-colonisateur. La première phrase du roman nous étonne par ses connotations figurées où l'auteur décrit la vie de son peuple autrefois et aujourd'hui, à travers sa description de sa relation illégale avec l'amante française Céline, pleine de débats et de querelles; au début du roman, Boudjedra fait allusion à la période de la présence du colonisateur en Algérie en la qualifiant aussi comme une période d'hallucinations où le

(11) Gafaïti (Hafid), Rachid Boudjedra, une poétique de la subversion, Autobiographie et Histoire, Op. Cit., p. 40.

(12) Dugas (Guy), "Approche socio-historique : une ou des littérature(s) maghrébine(s)" in Memmes, Abdallah, Approches scientifiques du texte maghrébin, Casablanca, Toubkal, 1987, p.72. 
peuple était trompé par le discours malin de l'Autre. Cette Française dont le narrateur est tombé amoureux et à laquelle il s'adresse en racontant tout au long du roman, cristallise bien la relation franco-algérienne qui ne se terminera jamais : "Avec la fin de l'hallucination venait la paix lumineuse (...) nous avions donc cessé nos algarades(...)."LR. ${ }^{(*)} p .1$; Boudjedra expose ses idées concernant cette relation incompréhensible entre l'Algérie et la France; le narrateur du roman et Céline la française ont été aussi qualifiés comme des : "boxeurs prêts non pas à se battre mais à se mordre jusqu'au sang." LR. p. 11; Le narrateurpersonnage poursuit sa description de cette relation ambiguë en disant : "le flou caractéristique de nos rapports" LR. p.12

Boudjedra a voulu montrer que l'indépendance du pays n'empêche pas le colonisateur d'être au courant de tout ce qui se passe dans sa colonie la plus riche; toujours, Céline demande au narrateur du roman de lui confier ses secrets les plus intimes : "Parle-moi encore de ta mère" LR., p.16;

À travers "La Répudiation", On constate bien la part idéologique de Boudjedra sur l'identité algérienne et ses composants les plus variés au long des siècles; une identité toujours en contact avec diverses civilisations dont la civilisation française était et reste encore l'une des plus importantes. Le narrateur-personnage parle toujours de sa relation troublante avec la femme-aimée française :"L'orage était sur le point d'éclater entre nous..." LR., p.17; cette relation étendue, même après le départ du colonisateur, entre Rachid, l'Algérien, et Céline, la Française, affirme que la colonisation a occupé également la mentalité des Algériens qui vivent encore en contact avec l'Autre qui ne pourrait pas oublier sa présence en Algérie et qui voudrait être au courant de tout ce qui se

(*) Nous avons choisi cette abréviation "LR." pour désigner le roman à étudier " La Répudiation" 
déroule après son départ :" Céline me demandait de reprendre le récit que j'avais abandonné la veille..." LR., p.11; "Je passais mes journées à raconter la vie de la tribu, la mort de Zahir,(...) "Continue à évoquer la maison de Ma", disait-elle." LR., pp.233-234

Boudjedra reflète la situation des Algériens avant et après l'indépendance ; tout au long du roman, Rachid est menacé et ne se sent pas à l'aise malgré sa présence dans son pays d'origine et malgré le départ du colonisateur. La peur du narrateur et son inquiétude reviennent alors à la présence d'un appareil sécuritaire en Algérie, établi après l'indépendance, qui représente une source de terreur pour lui et bien sûr pour tous les Algériens : "Je savais que les Membres Secrets étaient à l'affût de la moindre incartade pour m'envoyer, cette fois-ci définitivement, au bagne." LR., p.31

Boudjedra donne beaucoup d'importance à cette date dans l'Histoire de l'Algérie " 8 mai 1945", où les Algériens commencent à se révolter contre l'oppression du colonisateur. Le narrateur-personnage cite également le nom d'une prison nommée Barberousse ${ }^{(\bullet)}$ et ce que font les Français avec les femmes :" Barberousse, prison d'une rondeur sacerdotale qui semble prolonger exprès l'attente des femmes qui font continuellement la queue depuis le 8 mai 1945, passant devant les gardiens corses qui les fouillent." LR., p.80 Charles-Robert Ageron apprécie que :" Les "événements de mai 1945 » commencèrent en effet le ler mai puis le 8 mai un mouvement insurrectionnel local éclata à Sétif et à Guelma..." (13); en traitant la question de la révolte des Algériens durant la période

$\left(^{\bullet}\right.$ ) Prison de Barberousse, nom d'une des prisons d'Alger construite lors de la période coloniale.

(13) Ageron (Charles-Robert), Mai 1945 en Algérie : Enjeu de mémoire et histoire, in Matériaux pour l'histoire de notre temps, 2012/4, $\mathrm{N}^{\circ} 108$, p. 69 
coloniale, Boudjedra jette quelque lumière sur la participation de toutes les catégories sociales, même les lycéens ont exprimé leurs points de vue en classe. Le narrateur-personnage parle de sa situation comme lycéen dépourvu de tous ses droits dans son pays à cause de l'administration de l'école appliquant les ordres du régime établi, il explique que: "C'était en fait une action politique que nous cherchions à travers les cours de poétique arabe: nous voulions créer des incidents et provoquer l'administration, hostile à nos activités nationalistes." LR., p.182 ; ainsi, Boudjedra dévoile le rôle de l'enseignement dans la renaissance des nations.

A travers le narrateur-personnage du roman, Boudjedra révèle l'un des lieux haïssables pour tout être humain : c'est la prison. En feuilletant "La Répudiation", on découvre que la vie de Rachid était divisée entre deux lieux principaux : la prison et l'hôpital, Rachid dit :" j'avais pleinement conscience d'avoir fait la navette entre le clan et le bagne, puis entre le bagne et l'hôpital" LR., p. 233 ; il est remarquable que la prison occupe une grande partie dans la mentalité du personnage. Ici, Boudjedra nous fait rappelle la prison de Lambèse ${ }^{(\mathbf{})}$ qui est considérée comme l'une des plus anciennes prisons dans l'Histoire contemporaine de l'Algérie. L'appellation de Lambèse a été transformée actuellement en Tazoult :" Lambèse devenue Tazoult a été transformée au XIX siècle en colonie pénitentiaire, assez réputée pour devenir proverbiale.» ${ }^{(14)}$

Ainsi, Boudjedra a mis nu les conditions difficiles dans lesquelles ont vécu les Algériens durant et après la période coloniale en dévoilant que la souffrance de son peuple n'a pas

(") C'est une ville militaire d'Afrique romaine se situant au nord-est de l'Algérie sur le territoire de la commune de Tazoult dans la région des Aurès, à $10 \mathrm{~km}$ à l'est de Batna, sur la route de Timgad et de Khenchela.

(14) https://www.cairn.info/revue-histoire-urbaine-2007-2-page-166.htm, p.167 
encore terminé ; les prisons inhumaines restent encore ouvertes. Lambèse était le grand témoin, elle a été employée avant et après l'indépendance par le colonisateur et aussi par le pouvoir établi. Ce bagne sert à emprisonner tout opposant au pouvoir ; Rachid nous souligne la situation en quelques lignes : "Je soupçonnais le Clan de m'avoir enfermé, avec l'accord de mon père, dans le bagne de Lambèse, en même temps qu'un grand nombre de détenus politiques qui y moisissaient depuis de longues années, sans avoir jamais été jugés ni même informés des charges retenues contre eux." LR., p.239.

Mis en majuscule la première lettre du mot Clan dans la citation précédente, Boudjedra fait allusion au Clan Oujda qui représente : "un groupe politico-militaire composé d'algériens $d u$ Front de libération nationale (FLN) durant la guerre d'Algérie. ${ }^{\prime \prime(15)}$; Mais son activité politique a effectivement commencé à partir de l'indépendance du pays, "Une partie des présidents successifs de l'Algérie qui ont gouverné le pays plus de dix années, sont issus de ce Clan depuis le coup d'État du 19 juin 1965, (Houari BOUMEDIENE, Chadli BENDJEDID et Abdelaziz BOUTEFLIKA). "(16)

À travers l'histoire fictive de la présence d'un fotus dans l'utérus de Zoubida, la marâtre du narrateur, Boudjedra a voulu exprimer son espoir de voir renaître un nouveau pays après tous ces malheurs. Zoubida souligne peut-être la naissance d'une Algérie nouvelle portant la vraie révolution dans ses entrailles. La déception de Boudjedra s'annonce finalement en exprimant que ce fotus est un mythe, il n'existe pas :" Le foetus n'était pas l'enfant de la marâtre-amante, mais le pays ravalé à une goutte de sang gonflée au niveau de l'embryon puis tombée en désuétude dans une attente prosternée de la violence qui tardait

(15) https://jeanyvesthorrignac.fr/wa_files/info_508_oujda.pdf (16) Loc. Cit. 
à venir." LR., p.241; Armelle Crouzières-Ingenthron apprécie que :" Le foetus est bien mort ou n'est jamais né car la vraie révolution tant attendue et espérée n'a jamais eu lieu. Nous sommes en "non-révolution". (17)

Dans les dernières pages du roman, Boudjedra s'adresse à son peuple ou plutôt au régime politique qui gouverne le pays, en posant des questions concernant la situation politique de l'Algérie, il emploie aussi l'adjectif possessif "notre" qui réfère à la première personne du pluriel pour affirmer que sa voix est plurielle :" Notre armée n'était-elle pas la plus puissante du Maghreb? N'étions-nous pas membres influents de l'O.N.U. ?" LR., p. 248

"La Répudiation" est un roman socio-politique qui exprime bien une part très importante de l'aspect politique chez Boudjedra qui ne tarde pas à donner un conseil aux responsables du pays en souhaitant un bon avenir à l'Algérie. Ainsi, le narrateurpersonnage dit: "Il faudrait fermer toutes les geôles du pays et les transformer en écoles du soir pour les éventuels chômeurs qui, par quelque miracle extraordinaire, existeraient encore en dépit des efforts planifiés du régime et de sa confiance dans l'investissement humain." LR., p.247

\section{L'aspect érotique :}

Boudjedra, comme écrivain algérien musulman, a traité dans son premier ouvrage romanesque "La Répudiation" des thèmes qui pourraient choquer le lecteur arabo-musulman, des thèmes difficiles de toucher dans un pays où l'islam est la religion officielle et les traditions arabo-islamiques s'imposent. Ce roman n'a pas rencontré de réussite remarquable chez le lecteur algérien, du fait que le romancier a exprimé ses points de vue

(17) Crouzières-Ingenthron (Armelle), Le double pluriel dans les romans de

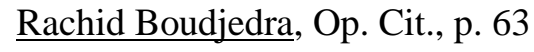


loin de toute règle de bienséance. Boudjedra a le courage d'insérer dans son récit des mots et des phrases qui blessent la pudeur de tout arabe musulman. Boudjedra a bien montré une part très importante du tabou non pas seulement chez la femme, mais aussi chez l'homme. L'essentiel chez l'écrivain de "La

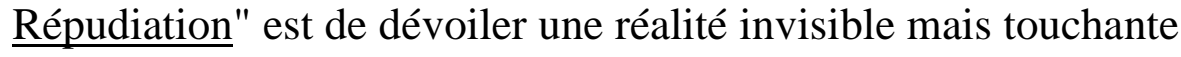
dans sa société. Il a choisi d'être un écrivain libérateur pour démasquer quelques réalités bien ancrées. Marc Boutet de Monvel apprécie que :" La notoriété des romans de Boudjedra et leur force de provocation tient pourtant d'abord à leur transgression insistante des tabous biffant le corps camouflé, castré, dans la société arabo-musulmane". ${ }^{(18)}$

Le tabou chez Boudjedra s'est étendu pour frapper la plupart des personnages du roman dont la relation était énigmatique. Voyons la relation entre la mère répudiée et ses deux fils ; c'est une relation bizarre qui regroupe à la fois l'amour excessif pour la mère, qui a dépassé toutes les frontières et la voie par laquelle ils découvrent le monde caché de chaque femme. Le fils aîné Zahir raconte dans son carnet, découvert après sa mort, un épisode relatif aux menstrues de sa mère, choquant et incompréhensible pour un enfant :" Je vis pour la première fois du sang de femme; il coulait sur la cuisse de ma mère. Je crus en mourir (...) huit ans. Découverte, derrière la porte de la cuisine de chiffons imbibés (imprégné) de sang noirâtre." LR., p.104; À travers cet épisode, Boudjedra jette quelques lumières sur cette question concernant l'éducation sexuelle des enfants de la part des parents, considérée comme une chose taboue à toucher chez la plupart des familles arabes ; mais discuter ça avec l'enfant l'aide bien à comprendre son corps et être au courant graduellement de la nature de ses organes génitaux. Le romancier nous dévoile également une mère traditionnelle et

(18) Boutet de Monvel (Marc), Boudjedra l'insolé, L'Insolation, Racines et Greffes, éd. L'Harmattan, Paris, 1994, p.21 
ignorante qui, à travers ses comportements insensibles, cause des blessures psychiques chez ses enfants. Boudjedra nous donne un sous-titre émouvant à la fin de la page 25 du roman, "Lascivité des siestes méditerranéennes", à travers duquel le fils narrateur souligne la relation amoureuse entre son père et sa mère après le repas de déjeuner. Faire l'amour pour sa mère ressemble à un acte de meurtre :" Elle se déshabille en silence et très lentement, comme on va à l'échafaud." LR., pp. 34-35

Le fils poursuit sa description de cette union sexuelle en soulignant la supériorité des hommes sur les femmes: "Corps lourd et que la sieste rend plus lourd encore (...) la communication presque évidente devient bâclée (...) Il faudrait s'habiller et quitter la chambre. Le père dort déjà. " LR., p.35

Boudjedra affirme que la répudiation de la mère va participer non seulement à ruiner son état d'âme, mais aussi à bouleverser complètement le comportement et la nature des enfants. Ainsi, le fils-narrateur cherche à se venger de son père en faisant l'amour avec Zoubida, sa deuxième femme :" Je couchais donc avec la femme légitime de mon père "LR., p.120; MohammedSalah Zeliche apprécie que : "De fait, l'inceste est réponse à une répudiation. C'est pourquoi il est lui-même l'expression d'une rupture (celle d'avec un ordre) et l'aboutissement d'une haine irrépressible." ${ }^{\prime(19)}$

Boudjedra traite également la question de la masturbation des garçons lors de leur solitude. Ce qui pousse le fils-narrateur à pratiquer l'onanisme est aussi sa recherche dans la chambre des buanderies, il raconte que :" les culottes des cousines, souillées à l'endroit du sexe d'une traînée jaune dont la seule évocation me faisait bander. Premières masturbations dans la grande

(19)Zeliche (Mohammed-Salah), L'écriture de Rachid Boudjedra, Poét(h)ique des deux rives, éd. Karthala, Paris, 2005, p.60 
cour." LR., p. 44 ; "J'allais me masturber dans les toilettes" LR., p.103

Après le remariage du père et la répudiation de la mère, Rachid, le fils-narrateur, plonge dans le tabou :" Je forniquais avec les veuves et les divorcées." LR., p.66; il entre également dans une relation illégale avec Yamina qui :" sortit alors un sein banal de fillette précoce que je m'empressai de malaxer(...) la chaude mamelle minable (...) son téton dur et bleuâtre" LR., p.51

L'inceste va s'étendre également à la relation entre le frère et sa sœur juive Leïla, une fille illégale née d'une relation taboue. Le fils-narrateur était sur le point d'accomplir l'acte sexuel avec sa sœur : "Leïla, ma demi-sœur juive que j'avais failli violer un soir, dans une chambre de la maison de ma mère, alors qu'elle s'amusait à m'embrasser sur la bouche et à dénuder en ma présence sa poitrine splendide." LR., p. 240

Le fils-narrateur décrit également l'état malheureux qui frappe le corps de sa mère après avoir être répudiée par le père : "L'ovulation, chaque mois, se dégonflait lamentablement." LR., p.38; "Elle obtient l'orgasme solitairement, avec la main ou bien avec l'aide de Nana (*)." LR., p.93; peut-être c'est une invitation de la part du romancier à bien comprendre l'état d'âme de chaque femme répudiée.

Boudjedra traite également la question de l'homosexualité à travers le personnage de Zahir, qui est le frère aîné de Rachid et qui souffre également de la répudiation de sa mère en voulant prendre une revanche sur son père. Le fils-narrateur parle de son frère en disant :" Zahir, lui, n'aimait pas les femmes. Il était amoureux de son professeur de physique." LR., p.103 ; ce n'est pas seulement Zahir qui est décrit d'homosexualité, Rachid

( ) Nana a plusieurs sens " jeune fille - petite amie et aussi prostituée au sens péjoratif." (https://www.linternaute.fr/dictionnaire/fr/definition/nana/) 
affirme qu'elle est devenue un phénomène social :" pour arriver à la maison de Ma, il faudrait éviter les impasses urineusesrendez-vous d'homosexuels qui se caressent dans noir des latrines." LR., p.61

En analysant le personnage de Zahir, Mohammed-Salah Zeliche constate que: "L'inceste et l'homosexualité sont une échappatoire, un moyen d'échapper à son destin ${ }^{(20)}$; mais Juliette Minces nous donne une autre justification de pratiquer l'homosexualité par les hommes: "la pénurie des femmes disponibles, et la difficulté de réunir la somme destinée au douaire - toujours obligatoire - retarde souvent le mariage des hommes qui trouvent alors momentanément remède dans l'homosexualité » ${ }^{(21)}$

L'Algérie est un pays arabe musulman où l'homosexualité est interdite et celui qui est coupable de cet acte est puni par loi ; L'homosexualité est considérée comme : " un délit - passible d'une peine de deux mois à deux ans de prison et d'une forte amende."

Ainsi, Boudjedra a découvert quelques aspects de sa société concernant le côté sexuel légal ou illégal; il indique que la famille joue le premier rôle dans les comportements de ses enfants dans la société.

\section{L'aspect social :}

Rachid Boudjedra nous présente un roman traditionnel dont le thème principal se fonde sur la famille, et surtout la mère

(20) Zeliche (Mohammed-Salah), L'écriture de Rachid Boudjedra, Poét(h)ique des deux rives, Op. Cit., p.52

(21) Minces (Juliette), La femme dans le monde arabe, Paris, Mazarine, 1980 , p. 49

(22) https://www.france24.com/fr/20171009-homosexualite-algerie-tentenlgbt-mariage-rainbow-convenance 
répudiée. Toujours et à jamais c'est la femme qui est le pivot de la plupart des contes, sa présence dans les œuvres littéraires est bien sûr relative à celle dans notre vie quotidienne. Le romancier voit que la femme subite l'humiliation non seulement socialement mais aussi religieusement. La société et la religion participent à la souffrance de la femme algérienne, d'après "La Répudiation".

Le pivot central du roman se déroule autour d'une femme répudiée, négligée de la part de son mari. Il est clair que l'auteur n'a pas donné un nom à cette femme, et cela pour souligner sa perte d'identité à côté de son mari ; dans les sociétés arabes, sa présence en couple lui donne la force et la dignité ; mais sa répudiation l'a rendue marginalisée. Le statut féminin dont l'insécurité réside dans le titre du roman " La Répudiation" est toujours en danger tant que la femme reste traditionnelle et tant que la société reste ancestrale. Fouzia Benmerabet apprécie que:" Le récit boudjedrien est un amalgame d'événements détournés à chaque fois selon le délire conscient ou inconscient du narrateur personnage. Parmi tous ces événements racontés, le plus dominant qui submerge le récit est la répudiation de la mère." ${ }^{\prime(23)}$

À travers son roman, Boudjedra traite la souffrance de toutes les femmes algériennes; ainsi, le fils-narrateur décrit sa mère en disant qu'elle était : "une victime au même titre que les autres femmes du pays..." LR., p.13 ; Boudjedra constate que la femme algérienne en général déploie des efforts pour garder son mari auprès d'elle par tous les moyens possibles, le fils-narrateur montre l'attitude des femmes pour saluer leurs époux : "Femelles indécises, elles rivalisent d'ingéniosité pour garder le

(23) Benmerabet (Fouzia), L'espace du tragique ou le drame de la mère dans La Répudiation de Rachid Boudjedra, in Synergies Algérie, no 26, 2018, p. 90 
mari dont elles baisent encore la main, en signe de respect." LR., P.40

Le fils-narrateur poursuit sa description de sa mère dans une série de phrases négatives exclamatives montrant la faiblesse et la soumission de la femme auprès de son mari:" Aucune révolte! Aucune soumission! (...) Aucun droit!" LR., p.33 ; et tout cela revient à l'autorité inéluctable du père :" mon père ne permettrait aucune manifestation." LR., p.33

Le fils-narrateur éprouve une sensation particulière d'affection envers sa mère qu'il donne souvent l'appellatif "Ma" ou ma mère", qui expriment à quel point Rachid est très proche de sa mère et très tendre avec elle ; tandis qu'il s'adresse rarement à son père en utilisant "Mon père" p. 36, 67; le plus souvent les deux fils utilisent le prénom de leur père "Si Zoubir" p.30, 64, $69 . .$. etc. et aussi "le père" p. 39, 91, 97...etc. Rachid qualifie son père aussi par "le patriarche" p.41.

Ce n'est pas seulement la souffrance de la femme du fait de de la répudiation que Boudjedra met à nu, c'est aussi les effets néfastes de cet acte sur toute la famille ; le fils-narrateur décrit la famille après la répudiation de sa mère: "Après avoir répudié sa femme, il la mettait devant le fait accompli de son autorité permanente et, du même coup, il nous plaçait nous, ses enfants, dans une situation impossible. Entre nous, il disposait une barrière d'hostilité qu'il s'ingéniait à consolider. " LR., p. 41

En décrivant sa famille, le fils-narrateur souligne que :" Nous étions tous transpercés par la mort." LR., p.72; il a voulu affirmer la malédiction qui a frappé la famille après le remariage du père. Mariée toute jeune, la petite sœur Yamina, revenue à la maison de son père après avoir passée une expérience douloureuse du mariage, a trouvé la mort :" elle mourut de la couture (qu'elle haïssait) et de la fièvre intestinale. 
Elle n'avait que vingt et un ans." LR. p.139; "Zahir est mort depuis une éternité déjà." LR., p.135 ; Ainsi, on constate que la mort occupe aussi une place à part dans le roman à étudier. Giuliana Toso Rodinis apprécie que :"Boudjedra se propose(...) une société qui ne conduit qu'à l'hôpital psychiatrique ou à la mort $^{\prime \prime 24)}$

Il est à noter que le mariage du père représente la cause principale qui a poussé Zahir, le fils aîné, à se jeter dans les bars et à devenir alcoolique. Rachid nous explique :" Mon frère a dix-sept ans et fréquente les bars louches de la ville depuis la répudiation de ma mère. Il boit dans les bars espagnols, italiens et juifs de la ville." LR., p.101. L'alcoolisme est un danger qui menace tous les jeunes; ce n'est pas seulement le remariage du père qui a détruit Zahir, mais aussi l'absence de tout contrôle du père : "Zahir n'avait jamais eu de père." LR., p.153 ; Boudjedra adresse un message à tout père d'être attentif aux actes de ses enfants à travers le portrait de Zahir; il a voulu dévoiler l'autorisation de l'alcoolisme dans un pays arabe musulman. Le fils-narrateur décrit comment son frère a été mort :" Mourir ivre, quelle aubaine!" LR., p.171

Boudjedra traite également les fêtes de noces organisées par le père; en employant des mots très simples mais expressifs, il décrit la mère :" la mort sur le visage" LR., p.63; alors que le père "pouvait éclater de bonheur" LR., p.64. Et pour les sœurs $\mathrm{du}$ narrateur, "elles ne pouvaient participer au complot." LR., p.69; Boudjedra, à travers le remariage de Si Zoubir, aborde la question du mariage précoce pour les jeunes filles. Le filsnarrateur montre que l'écart d'âge entre la jeune fille mariée et son père est plus grand :" Noces drues. La mariée avait quinze ans. Mon père, cinquante." LR., p.64; le romancier a voulu

(24) Toso (Rodinis Giuliana), Fêtes et défaites d'éros dans l'œuvre de Rachid Boudjedra, éd. L'Harmattan, Paris, 1994, p.38 
souligner que ce mariage inapproprié remonte à la pauvreté de certaines familles qui sont obligées à payer leurs jeunes filles pour bien profiter financièrement. Cette affaire concerne toutes les familles arabes qui cherchent la fortune à travers le mariage de leurs filles mineures. Ainsi le fils-narrateur parle de sa bellemère : "Zoubida(...) venait d'une famille pauvre et le père n'avait certainement pas lésiné sur le prix." LR., p.64 ; "achetée à quinze ans par le père." LR., p.123 ; Zoubida, elle-même, raconte les circonstances de son mariage avec Si Zoubir en disant que ce mariage :" n'avait été que la conclusion d'une affaire financière." LR., p.124

Ainsi, Boudjedra s'intéresse aussi à montrer l'une des traditions maghrébines très anciennes: c'est interdit de grouper les femmes avec les hommes dans un seul lieu ; la promiscuité est défendue. En décrivant la fête du remariage de son père, le narrateur dit :" pendant la noce, les femmes étaient séparées des hommes "LR., p.64

Il est à noter que Boudjedra jette quelques lumières sur un phénomène très répandu dans tout le monde : c'est la mendicité à laquelle Boudjedra donne une place dans son premier ouvrage romanesque. Notre romancier, comme écrivain algérien, touche la plupart des phénomènes qui frappent son pays d'origine. Il traite la mendicité dès les premières pages du roman, le filsnarrateur nous dit que : "Dehors, nous avions quand même peur des mendiants acharnés à notre poursuite auprès des étrangers en visite dans la ville." LR., p.22 ; "les mendiants qui infestent la ville" LR., p.48

Le fils-narrateur souligne que durant la fête des noces de son père, il y avait des mendiants; mais ils étaient présents en dehors de la maison. Rachid les qualifie ainsi :" Les poubelles, devant la maison, étaient prises d'assaut par les mendiants handicapés physiquement(...) la plupart étaient paralytiques, se 
traînaient à quatre pattes(...) Les aveugles, eux, surgissaient plus tard..." LR., pp.69-70. Boudjedra indique également la présence d'un autre type de mendicité, ce sont celles qui demandent l'aumône et sont prêtes à faire l'amour aussi pour obtenir de l'argent. Le fils-narrateur dit que :" En bas, dans la rue, les vieilles mendiantes arrivent déjà(...) Le maître ne fait rien pour chasser les mendiantes (...) il leur fait toujours des propositions malhonnêtes et elles les acceptent, à condition d'être payées." LR., p.95

Rachid Boudjedra traite aussi la question de la sorcellerie, très répandue au Maghreb, c'est à travers la personnalité de la mère du narrateur qu'il jette beaucoup de lumières sur ce phénomène. Cette mère est analphabète, elle ne sait ni lire ni écrire; mais elle est "sorcière" LR., p.86; c'est-à-dire qu'elle se livre à des pratiques de sorcellerie. Le fils-narrateur indique que: "d'un nègre que nous allions consulter ma mère et moi, en cachette du père, et qui entrait en transe en se serrant la tête avec un fichu multicolore(...) en promettant le retour du mari perdu" LR., p. 133. J.-H. Probst-Biraben constate, d'une part, la continuité des vieilles traditions chez les Arabo-berbères du nord de l'Afrique, et en particulier en Algérie où se sont superposées des croyances diverses apportées par les Nègres ; et, d'autre part, la présence des esclaves des musulmans dont les pratiques religieuses, purement mystiques à leurs débuts, ont été infectées de sorcellerie et de magie par les Nègres. ${ }^{(25)}$

(25) Cf., Probst-Biraben (J.-H.), Le Serpent, persistance de son culte dans l'Afrique du Nord, in Journal de la Société des Africanistes, 1933, tome 3, fascicule 2, p. 289. 
Mehdi Isikioune estime que :" Le phénomène de la sorcellerie et la profanation des tombes est devenu, ces dernières années, un phénomène en vogue en Algérie. ${ }^{\prime 26)}$

Boudjedra affirme que l'absence de prise de conscience est la cause de tous les maux qui frappent les hommes; ainsi il décrit la société : " aucune conscience sociale! Tout pourrissait, jutait... Puanteur." LR., p.69

En écrivant "La Répudiation", Rachid Boudjedra s'occupe de présenter un portrait sincère de tout ce qui se rattache à la société dans laquelle il vit, il souhaite changer la mentalité de ses compatriotes vivant encore dans l'ignorance et dans les traditions des ancêtres.

\section{L'aspect religieux :}

L'aspect religieux apparaît clairement dans le roman de Boudjedra où il ne tarde pas à traiter la religion comme l'argumentation valable du père pour répudier son épouse et se remarier avec une autre. Giuliana Toso Rodinis estime que : "On s'aperçoit que la narration est organisée autour d'un noyau central, la répudiation légalisée de la femme..." (27)

Alors la question des droits de la femme est toujours une source de polémiques dans toutes les religions. Boudjedra, comme écrivain musulman, critique la condition déplorable de la femme dans son pays d'origine, il appartient à ceux qui croient que la femme est encore privée de ses droits; Zeina el Tibi

(26) Isikioune (Mehdi), La sorcellerie fait des ravages en Algérie : Des cimetières entiers transformés en laboratoire de charlatanisme, in Le courrier d'Algérie, disponible sur " https://lecourrier-dalgerie.com/lasorcellerie-fait-des-ravages-en-algerie-des-cimetieres-entiers-transformesen-laboratoire-de-charlatanisme/"

(27) Toso Rodinis (Giuliana), Fêtes et défaites d'éros dans l'œuvre de Rachid Boudjedra, Op. Cit., p.30 
apprécie que :" Aux yeux de certains, l'islam serait la religion qui opprime les femmes; la femme musulmane vivrait en marge de la société, dans une situation d'infériorité, dominée par l'homme et empêchée d'évoluer. ${ }^{\prime(28)}$; nous sommes alors devant un problème socioreligieux concernant la femme et ses droits, il est remarquable que les livres saints comme la Bible, l'Évangile et le Coran rendent hommage à la femme. On trouve que la vierge Marie est mentionnée plusieurs fois au Coran et on a aussi une sourate complète titrée "Maryam" et cela est considéré comme une consécration de la femme dont la présence est inévitable pour que la vie continue. Zeina el Tibi constate qu': "il faut répéter que l'islam a donné à la femme un statut qui la respecte et l'honore, tant sur le plan spirituel que sur le plan social." ${ }^{\prime(29)}$ À travers "La Répudiation", Boudjedra, lui-même, cherche vainement la place perdue de la femme dans une société masculine :"Les hommes ont tous les droits, entre autres celui de répudier leurs femmes." LR., p.34 C'est à travers cet acte de répudiation que la société condamne les femmes. Ce n'est pas la religion qui fait souffrir les femmes, c'est la société des mâles qui appliquent la religion à leur guise, et aussi les sociétés basées sur des traditions et des coutumes très éloignées des préceptes religieux.

Boudjedra donne beaucoup d'importance au mois de Ramadhan, considéré comme le mois le plus sacré chez les musulmans. C'est le mois du jeûne, l'une des cinq obligations de l'islam. Ce mois suit un calendrier spécifique basé sur les cycles lunaires. Le fils-narrateur souligne l'arrivée de Ramadhan :" Nous savions guetter la lune" LR., p.19; son importance religieuse apparaît clairement à travers les événements du roman où le fils-

(28) El Tibi ( Zeina), La place de la femme dans l'islam, in Société, droit et religion", numéro 4, 2014, p.59

(29) El Tibi ( Zeina), La place de la femme dans l'islam, in Société, droit et religion", Op. Cit., p.60 
narrateur souligne l'impact spirituel de l'arrivée de ce mois : "Zahir s'arrêtait de boire pendant un mois. Ma reprenait espoir. La maison avait un air de fête(...) Paix tacite avec les oncles(...) le père nous laissait tranquilles." LR. p.19

Boudjedra aborde également un lieu sacré, privilégié chez les musulmans : c'est la mosquée où se regroupent les musulmans, surtout au Ramadhan :" Auparavant, nous allions à la mosquée(...) Ferveur réelle. Rangs de fidèles, mais les femmes derrière les hommes, au fond de la mosquée (...Voix mélodique de l'imam(...) Coran." LR., p.20

Boudjedra montre également à son lecteur qu'il y a des catégories des gens qui sont dispensés de jeûne, parmi lesquelles il cite les jeunes filles ou les femmes ayant ses règles. En parlant des femmes pendant le mois de Ramadhan, Rachid informe qu':" elles ne jeûnent pas, à cause de leurs règles mensuelles." LR., p.24 ; à travers le personnage de Zahir, le romancier exprime sa compassion pour les femmes qui souffrent des règles, peut-être cette pitié est une sorte de révolte contre cette injustice divine qui frappe les femmes en les rendant privées d'accomplir le jeûne d'un côté, et subies à des souffrances physiques insupportables. Zahir décrit les femmes comme :" porteuses du sang" LR., p.25

Mohammed-salah Zeliche apprécie que : "Zahir, le frère aîné, aimait bien sa sœur Saïda. Mais celle-ci est femme, elle pue les règles dont elle est condamnée à être le siège plusieurs jours tous les mois; elle est dotée d'un sexe qui ne symbolise pas autre chose qu'une blessure et l'endroit d'une douleur. Etre de souffrance, de faiblesse, de déchéance, damné par une justice divine mais arbitraire, désarmé et résigné, elle communique à 
son frère sa désespérance et devient à la fois objet d'attirance et de répulsion." $\left.{ }^{30}\right)$

Boudjedra souligne également que les jeunes filles musulmanes doivent porter le voile à un certain âge ; le fils-narrateur indique que :" elles avaient dépassé l'âge de la puberté et annonçaient des poitrines splendides. Elles allaient au lycée mais portaient le voile." LR., p. 85 ; tandis que dans "le quartier juif. Les femmes ne portent pas le voile." LR., p.205

Boudjedra affirme la présence forte des juifs en Algérie; en parlant de son allée au lycée, Rachid mentionne : " le professeur juif qui (...) enseignait le français." LR., p. 54 ; Zahir, dans son carnet, raconte sa relation étroite avec son professeur athée de physique qui se connaît comme "Heimaltos" LR., p. 103 ; "ce juif athée prétend que la Bible est le plus beau poème que les hommes aient jamais écrit; j'ai freiné son enthousiasme en soutenant que le Coran était beaucoup plus beau." LR., p.105 ; Heimaltos, après l'annonce de la mort de Zahir, est revenu d'Israël et allé rester dans la chambre de Rachid en attendant l'arrivée du cadavre de son ami ; le fils-narrateur souligne que sa mère n'accepte pas "la présence du juif dans la maison mortuaire." LR., p. 154 ; mais cela n'empêche pas ce juif de se plonger :" avidement dans la lecture de la Bible." LR., p. 155 ; alors que Zahir est en amitié avec le juif, la mère "maudissait les juifs(...) la mère, sitôt le professeur parti, aérait les chambres, lavait les verres dans lesquels le mécréant avait bu et récitait des formules incantatoires." LR., p. 104 ; à travers le comportement de la mère envers le juif, Mohammed-Salah

(30) Zeliche (Mohammed-Salah), L'écriture de Rachid Boudjedra, Poét(h)ique des deux rives, Op. Cit., p.56 
Zeliche constate que Boudjedra :" met à nu le racisme juif antiarabe en réaction au racisme arabe anti-juif et vice versa." ${ }^{\text {(31) }}$

Comme écrivain musulman, Boudjedra n'hésite pas à retracer la vie religieuse dans son pays d'origine, la coexistence entre les musulmans et les juifs, en se concentrant sur quelques pratiques religieuses relatives toutes au sang, comme l'immolation de l'Aïd qui représente pour le narrateur "l'épreuve la plus terrifiante (...) la cérémonie durant laquelle on tuait plusieurs bêtes, pour perpétuer le sacrifice d'un prophète prêt à tuer son fils pour sauver son âme." LR., p. 194

Un autre point très remarquable dans le roman, c'est l'attachement de la mère du narrateur aux marabouts. Cette femme ignorante a beaucoup recours aux saints, et cela représente une réalité vécue par la plupart des gens de l'Algérie. Cette manière de pensée est nommée de la part de Leïla Babès "L'islam de la colonisation" qui est :" caractérisé par une multitude de saints et de groupements confrériques, que l'Occident colonial découvre, notamment en Algérie, au début du dix-neuvième siècle. ${ }^{\prime(32)}$; Rachid nous parle de sa ville en disant :" Dans notre ville les marabouts se multiplient." LR., p.93

A travers la parole du narrateur, Boudjedra souligne que le recours aux saints est devenu un phénomène dans tout le Maghreb : "Sidi Amor, un marabout de Tunis, célèbre dans tout le Maghreb(...) il n'y a que des femmes autour de lui." pp.109110; Boudjedra décrit également le marabout pour souligner la superficialité et la frivolité de cette société accablée par le poids

(31) Zeliche (Mohammed-Salah), L'écriture de Rachid Boudjedra, Poét(h)ique des deux rives, Op. Cit., p. 98

(32) Babès (Leiila), L'islam pluriel au Maghreb, in International Review of Community Development / Revue internationale d'action communautaire, numéro 26, 1991, p. 123 (https://doi.org/10.7202/1033898ar) 
colonial du passé et l'ignorance du présent; le fils-narrateur s'accompagne sa mère à Tunis pour rendre visite à Sidi Amor : "Le marabout n'est qu'un paralytique général (...) il n'y a que des femmes autour de lui." LR., pp.109-110

\section{L'aspect romanesque :}

Nous ne pouvons passer en silence la part romanesque utilisée par Boudjedra dans son premier ouvrage romanesque. Le romancier algérien se distingue toujours de son écriture singulière qui représente un mélange entre le réel et la fiction ; mais la vision réaliste domine, ce romancier expose franchement la réalité du pays longtemps capturé en cherchant la délivrance.

Ses romans ne suivent pas la technique habituelle du roman maghrébin; pour cela, Bahia Nadia Ouhibi-Ghassoul estime que :" dans les romans de Boudjedra, on ne suit plus "l'écriture d'une aventure mais l'aventure de l'écriture."(33); elle le décrit également comme un "théoricien, théoricien d'un genre nouveau.." $\left.{ }^{34}\right)$. Boudjedra a réussi à présenter une structure romanesque bien différente, c'est une structure découpée par la multiplicité des récits racontés. Ce roman s'approche du Nouveau Roman où l'absence de toute chronologie, et l'absence de l'intrigue. Aussi, en donnant à l'amante française le prénom de Céline : "Céline riait" LR., p.18, Boudjedra annonce son attachement à l'un des romanciers français du XX ${ }^{\text {ème }}$ siècle, c'est Louis Ferdinand Céline dont l'écriture se distingue des autres et dont le style est elliptique.

(33) Ouhibi-Ghassoul (Bahia Nadia), L'écriture dans l'œuvre de Boudjedra, in Rachid Boudjedra, une poétique de la subversion, éd. L'Harmattan, Paris, 1999, p.81

(34) Loc. Cit. 
Le style adopté par Boudjedra nous étonne et nous pousse à vérifier ses caractéristiques les plus étonnantes. En décrivant l'écriture de ce romancier et son style dans pas mal des romans, parmi lesquels figure "La Répudiation", Hafid Gafaiti apprécie que ses romans sont "caractérisés par un style baroque et une écriture marquée par l'excès et le déroulement infini du verbe." (35)

On remarque bien que les phrases de Boudjedra sont courtes "des phrases nominales" ; dans "La Répudiation", on rencontre souvent une phrase formée d'un seul mot; le romancier a bien sûr voulu attirer notre attention sur l'intérêt et la signification que porte chaque mot dans le roman. Citons par exemple, le mot "Ripailles." LR., p.19, écrit seul pour souligner comment les musulmans préparent leurs tables au mois de Ramadhan; il nous donne aussi dans le même contexte le mot "Foules." LR., p.20 pour décrire les rues durant la nuit du Ramadhan, après le repas de dîner.

Boudjedra utilise encore des mots simples pour décrire la souffrance de la mère du narrateur :" Solitude, ma mère! Fermeture!" LR., p.37; on a la sensation que le romancier s'adresse ici à la solitude comme une personne pour indiquer l'union inséparable entre la femme mère et la solitude dans laquelle elle vit ; et aussi le mot "Fermeture" souligne que toutes les portes sont fermées en face de la femme, elle est privée de toute liberté.

Il est nécessaire d'aborder le choix de Boudjedra d'écrire en utilisant la langue de l'Autre, qui a une grande prédilection chez lui. Cette question touche bien tous les écrivains maghrébins d'expression française, qui, à leur tour, ne tardent pas à justifier

(35) Gafaïti (Hafid), Rachid Boudjedra, une poétique de la subversion, Autobiographie et Histoire, Op. Cit., 1999, p. 41 
le fait d'écrire en français. Lors d'une conférence sur le bilinguisme, l'écrivain traite cette question toujours inquiétante de son choix de la langue française pour s'exprimer pour la première fois :" Mon désir le plus profond, c'était d'écrire en arabe au départ, mais il est certain qu'avec un roman qui était dans ma tête depuis l'enfance, La Répudiation, je ne pouvais pas penser une minute pouvoir publier ce livre en arabe dans un pays arabe en 1969." (36). Bien que ce choix lui donne la possibilité de s'exprimer franchement et l'occasion d'être connu autant en France qu'au Maghreb; Boudjedra considère la francophonie "comme une forme de néo-colonialisme." (37); Boudjedra déclare également que :" Pour moi, Algérien, je n'ai pas choisi le français. Il m'a choisi, ou plutôt il s'est imposé à moi à travers des siècles de sang et de larmes et à travers l'histoire douloureuse de la longue nuit coloniale." (38)

Armelle Crouzières-Ingenthron apprécie que : "Boudjedra a renouvelé la forme narrative du roman." (39)

En guise de conclusion, il est à noter que " La Répudiation" a bien indiqué tout ce qui se déroule dans la mentalité de Rachid Boudjedra. En tant que son premier ouvrage romanesque, ce roman a dévoilé la part idéologique d'un homme arabomusulman qui est devenu, du fait de la colonisation, un écrivain bilingue très attaché à la langue de l'Autre pour être capable de toucher l'interdit voire le tabou de la part de sa société. "La Répudiation" incite la curiosité du lecteur à découvrir les idées et les croyances de son écrivain. En abordant un thème social à

(36) http://miflc.com/wp-content/uploads/2020/06/CrouzieresIngenthronVolume-4.pdf, p.19

(37) http://miflc.com/wp-content/uploads/2020/06/CrouzieresIngenthronVolume-4.pdf, p.21

(38) Boudjedra (Rachid), Lettres algériennes, Paris, Grasset, 1995, p.30

(39) Crouzières-Ingenthron (Armelle), Le double pluriel dans les romans de Rachid Boudjedra, Op. Cit., p.126 
plusieurs visages, Boudjedra excelle à faire découvrir la face invisible de la société algérienne. Le romancier algérien souligne la souffrance de toute la famille après la répudiation de la mère et le remariage du père. L'écrivain a voulu indiquer également que pour terminer le mariage forcé entre la France et l'Algérie; il faudrait des victimes et du sang. La femme répudiée, c'est l'Algérie colonisée; et la nouvelle épouse, Zoubida, l'indépendance trompeuse. Pour ainsi dire, "La Répudiation" donne une vision politique, sociale, érotique et religieuse de Boudjedra ; et tout cela est formulé dans une vision romanesque bien distinguée.

\section{Bibliographie :}

\section{Le roman étudié :}

- La Répudiation, éditions Denoël, Collection Folio, Paris, 1969, 252 pages.

\section{Autres ouvrages cités de Rachid Boudjedra :}

- La Prise de Gibraltar, roman traduit de l'arabe par Antoine Moussali en collaboration avec l'auteur, éditions Denoël, Paris, 1987.

- Lettres Algériennes, éditions Grasset, Paris, 1995

Ouvrages consacrés partiellement ou totalement à Rachid Boudjedra ou à la littérature maghrébine d'expression française :

- Alemdjrodo (Kangni), Rachid Boudjedra, la passion de l'intertexte, éd. Presses Universitaires de Bordeaux, 2001, disponible sur le site :

https://books.openedition.org/pub/3086\#text 
- Boutet de Monvel (Marc), Boudjedra l'insolé, L'Insolation, Racines et Greffes, éd. L'Harmattan, Paris, 1994.

- Crouzières-Ingenthron (Armelle), Le double pluriel dans les romans de Rachid Boudjedra, L'Harmattan, paris, 2001.

- Gafaïti (Hafid), Rachid Boudjedra, une poétique de la subversion, Autobiographie et Histoire, éd. L'Harmattan, Paris, 1999.

- Minces (Juliette), La femme dans le monde arabe, Paris, Mazarine, 1980.

- Toso (Rodinis Giuliana), Fêtes et défaites d'éros dans l'œuvre de Rachid Boudjedra, éd. L'Harmattan, Paris, 1994.

- Zeliche (Mohammed-Salah), L'écriture de Rachid Boudjedra, Poét(h)ique des deux rives, éd. Karthala, Paris, 2005.

\section{Thèses consultées :}

- Achheb (Loubna), Quête de soi et désillusion dans L'escargot entêté de Rachid Boudjedra, L'Amour, la fantasia d'Assia Djebar et Le fleuve détourné de Rachid Mimouni, thèse de doctorat Présentée à la Faculté des Lettres et des Langues, Université SETIF 2, 2014.

- Ghadie (Heba Alah), Rachid Boudjedra autotraducteur, Thèse soumise à la Faculté des études supérieures et postdoctorales dans le cadre des exigences du programme de maîtrise en Lettres françaises, Département de français, Faculté des Arts, Université d'Ottawa, Canada, 2008.

- Radouane (Aissani), L'écriture plurielle dans l'œuvre de Rachid Boudjedra, thèse de doctorat, Faculté des Lettres 
et des langues, Université les Frères Mentouri Constantine 1, 2008.

\section{Articles et périodiques :}

- Ageron (Charles-Robert), Mai 1945 en Algérie : Enjeu de mémoire et histoire, in Matériaux pour l'histoire de notre temps, 2012/4, $\mathrm{N}^{\circ} 108$

- Babès (Leïla), L'islam pluriel au Maghreb, in International Review of Community Development / Revue internationale d'action communautaire, numéro 26, 1991, p. 123

(https://doi.org/10.7202/1033898ar)

- Benmerabet (Fouzia), L'espace du tragique ou le drame de la mère dans La Répudiation de Rachid Boudjedra, in Synergies Algérie, no 26, 2018

- Bonn (Charles), "La répudiation", ou le roman familial et l'écriture-espace tragique. In: Revue de l'Occident musulman et de la Méditerranée, n²2, 1976. pp. 175180

- Boudjedra (Rachid), in Présence Francophone, No. 19, Automne, 1979, pp.61-62

- Dugas (Guy), "Approche socio-historique : une ou des littérature(s) maghrébine(s)" in Memmes, Abdallah, Approches scientifiques du texte maghrébin, Casablanca, Toubkal, 1987

- Dumais (Monique), L'autre salut : femmes et religions. In Recherches féministes, Volume 3, numéro 2, 1990, p. 2 disponible sur https://doi.org/10.7202/057603ar

- El Tibi ( Zeina), La place de la femme dans l'islam, in Société, droit et religion", numéro 4, 2014

- Fouzia Benmerabet, L'espace du tragique ou le drame de la mère dans La Répudiation de Rachid Boudjedra, Synergies Algérie n² 26, 2018 
- Hamès (Constant), « Problématiques de la magiesorcellerie en islam et perspectives africaines », in Cahiers d'études africaines [En ligne], 189-190 | 2008, mis en ligne le 15 avril 2011, consulté le 19 avril 2019.

- Isikioune (Mehdi), La sorcellerie fait des ravages en Algérie : Des cimetières entiers transformés en laboratoire de charlatanisme, in Le courrier d'Algérie, disponible sur " https://lecourrier-dalgerie.com/lasorcellerie-fait-des-ravages-en-algerie-des-cimetieresentiers-transformes-en-laboratoire-de-charlatanisme/"

- Ouhibi-Ghassoul (Bahia Nadia), L'écriture dans l'œuvre de Boudjedra, in Rachid Boudjedra, une poétique de la subversion, éd. L'Harmattan, Paris, 1999

- Probst-Biraben (J.-H.), Le Serpent, persistance de son culte dans l'Afrique du Nord.. In: Journal de la Société des Africanistes, 1933, tome 3, fascicule 2.

\section{Sitobiographie :}

- https://www.cnrtl.fr/definition/id\%C3\%A9ologie

- https://www.linternaute.fr/dictionnaire/fr/definition/ideologie/

- https://www.cairn.info/revue-histoire-urbaine-2007-2-page$\underline{166 . h t m}$

- https://jeanyvesthorrignac.fr/wa_files/info_508_oujda.pdf

- https://jeanyvesthorrignac.fr/wa files/info_508_oujda.pdf

- https://www.france24.com/fr/20171009-homosexualitealgerie-tenten-lgbt-mariage-rainbow-convenance

- https://www.cairn.info/revue-sud-nord-2001-1-page-27.htm

- https://www.cairn.info/revue-politique-africaine-2016-2-page$\underline{169 . \mathrm{htm}}$

- https://journals.openedition.org/anneemaghreb/4458 
الجوانب الأيدولوجية في رواية " الطلاق " لرشيد بوجيدره

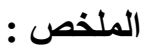

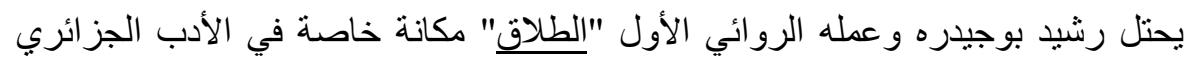

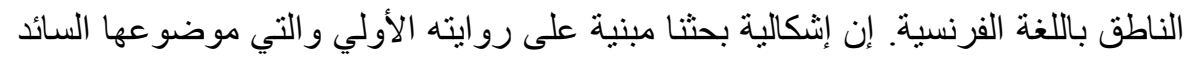

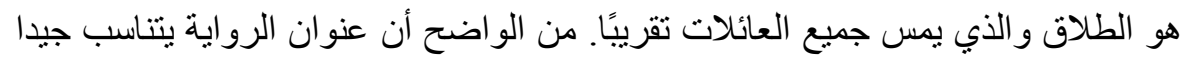
مع محتو اها.

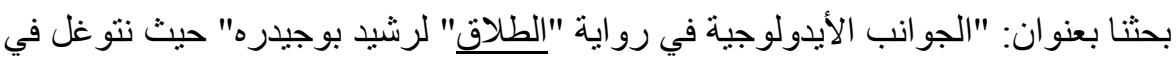

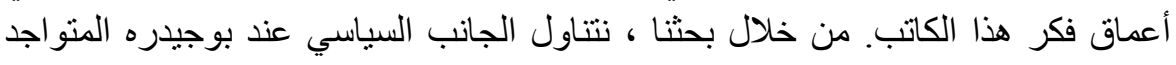

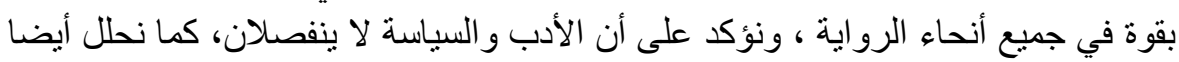

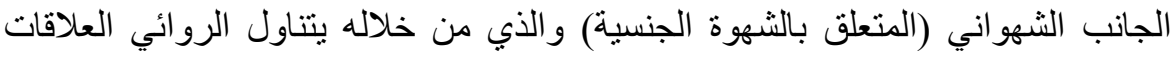

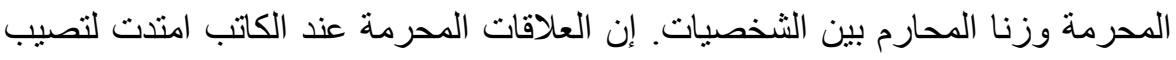

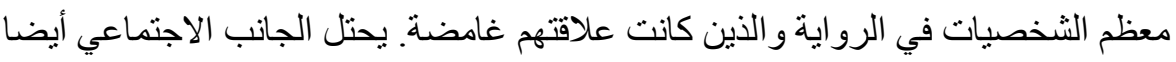

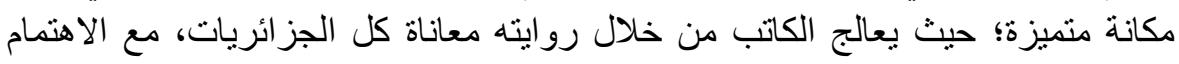

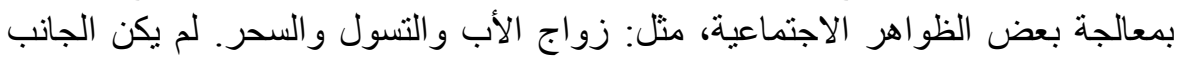

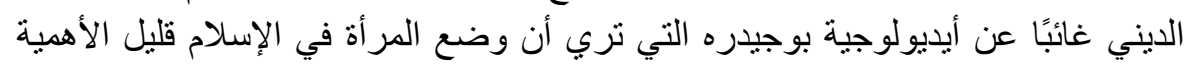

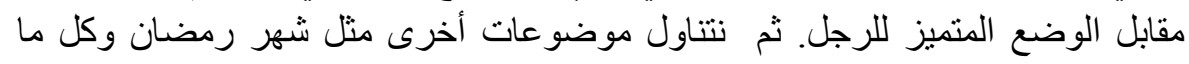

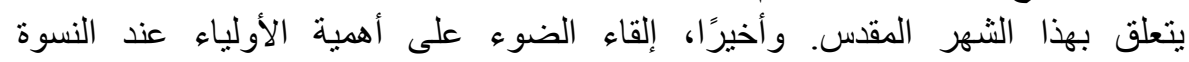

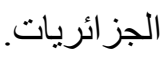

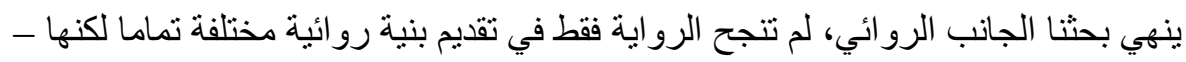

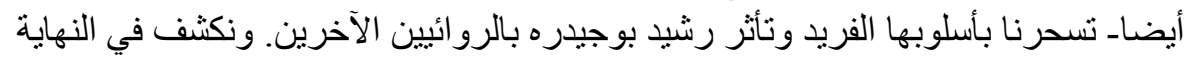
موضوع اختيار الكاتب للغة الآخر.

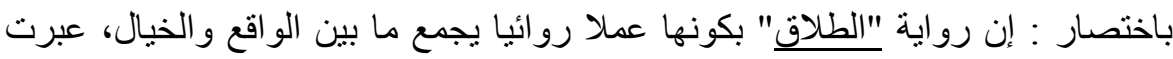

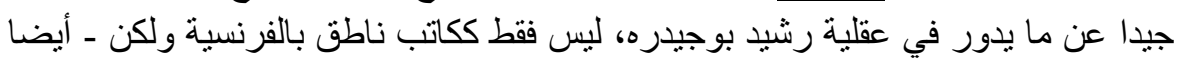

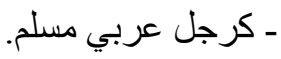

